\title{
Research Article \\ Fuzzy Variable Structure Control for Uncertain Systems with Disturbance
}

\author{
Bo Wang, ${ }^{1,2}$ Peng Shi, ${ }^{3,4}$ Hamid Reza Karimi, ${ }^{5}$ \\ Jun Wang, ${ }^{1}$ and Yongduan Song ${ }^{6}$ \\ ${ }^{1}$ School of Electrical and Information Engineering, Xihua University, Chengdu 610096, China \\ ${ }^{2}$ School of Applied Mathematics, University of Electronic Science and Technology of China, \\ Chengdu 610054, China \\ ${ }^{3}$ School of Electrical and Electronic Engineering, The University of Adelaide, Adelaide SA 5005, Australia \\ ${ }^{4}$ School of Engineering and Science, Victoria University, Melbourne, VIC 8001, Australia \\ ${ }^{5}$ Department of Engineering, Faculty of Engineering and Science, University of Agder, \\ 4898 Grimstad, Norway \\ ${ }^{6}$ School of Automation, Chongqing University, Chongqing 400044, China
}

Correspondence should be addressed to Peng Shi, peng.shi@vu.edu.au

Received 2 September 2012; Accepted 26 October 2012

Academic Editor: Mohammed Chadli

Copyright (C) 2012 Bo Wang et al. This is an open access article distributed under the Creative Commons Attribution License, which permits unrestricted use, distribution, and reproduction in any medium, provided the original work is properly cited.

This paper focuses on the fuzzy variable structure control for uncertain systems with disturbance. Specifically, the fuzzy control is introduced to estimate the control disturbance, the switching control is included to compensate for the approximation error, and they possess the characteristic of simpleness in design and effectiveness in attenuating the control chattering. Some typical numerical examples are presented to demonstrate the effectiveness and advantage of the fuzzy variable structure controller proposed.

\section{Introduction}

Since the pioneering works of Utkin in 1977 [1], the variable structure control (VSC) has generated considerable interests in control field. Up to now many researches on VSC have been carried out [2-16]. Based on VSC theory, [2] developed an adaptive fuzzy control system design method for uncertain Takagi-Sugeno fuzzy models with norm-bounded uncertainties. By using a high-gain observer, [3] presented an output feedback model-reference variable structure controller to achieve global exponential stability with respect to a small residual set without generating peaking in the control signal. In [4], the subordinated reachability of the sliding motion is introduced to realize the control on a class of uncertain stochastic 
systems with time-varying delay. Via introducing a pseudo-inversion, the authors in [5] discussed the adaptive control for the uncertain discrete time linear systems preceded by hysteresis nonlinearity. In [6], a sufficient condition for existence of reduced-order sliding mode dynamics was derived to realize the sliding mode control of a continuous-time switched stochastic system. For VSC, one of the most intriguing properties is the insensitivity to parameter uncertainties and external disturbance for the switching action between sliding modes, which can lead to the generation of chattering phenomenon and make a difference to system control performance. Therefore how to solve this problem is always a challenging topic for VSC.

Uncertainties and disturbances exist in many kinds of systems; this makes the practical control problem complicated and has received much attention from scholars [17-22]. VSC method is one of the effective solutions, and conventionally the switching term is built based on the upper norm bound of control disturbance to satisfy the system control condition. Therefore there exists the difference between real control disturbance and its upper norm bound. The maximum switching amplitude can be double disturbance error upper bound. For ease of use, the upper norm bound sometimes is taken as a constant by experience. This may lead to the serious chattering problem. Widely acknowledged, an effective solution is to build a unit to obtain the estimate of time-varying control disturbance. Up to now, there exist some feasible methods, such as neural networks and genetic algorithm, to tackle the problem. However in real application, those approaches are too complicated and need much more control information. Corresponding control cost problem cannot be ignored.

Recently, fuzzy method gets wide attention in the control field, corresponding research can be seen in [23-33] and the references therein. It is also introduced to VSC area for its characteristic of simpleness in design, and effectiveness in attenuating chattering. In this paper, a fuzzy auxiliary controller will be built to approximate the control disturbance based on just one feedback signal and a switching control term will be designed to compensate for the approximation error. Some typical simulation examples will be concerned afterward to illustrate the effectiveness of the controller given.

Notations used in this paper are fairly standard. Let $R^{n}$ be the $n$-dimensional Euclidean space, $R^{n \times m}$ represents the set of $n \times m$ real matrix, $(\cdot)^{(i)}$ denotes the $i$ th derivative of $(\cdot)$, and the notation $A>0$ means that $A$ is real symmetric and positive definite, $\operatorname{sgn}(x)$ denotes the operator $|x| / x$, and sat(.) denotes the saturated function.

\section{Problem Statement}

In this paper, the following high order uncertain single-input single-output (SISO) system with disturbance is considered:

$$
\begin{gathered}
\dot{x}_{i}(t)=x_{i+1}(t), \quad i<n, \\
\dot{x}_{n}(t)=f(x, t)+\Delta f(x, t)+p(t)+b(t) \cdot u(t),
\end{gathered}
$$

where $x(t)=\left(x_{1}(t), x_{2}(t), \ldots, x_{n}(t)\right)^{T} \in R^{n}$ is the system state vector, $f(\cdot, t)$ is the nonlinear function, $\Delta f(\cdot, t)$ is the nonlinear uncertainties, $p(t)$ is the external disturbance, $b(t)$ is the nonzero coefficient of control input, and $u(t)$ is the control input. 
Define the tracking error

$$
E(t)=x(t)-x_{r}(t),
$$

where $E=\left(e_{1}(t), e_{2}(t), \ldots, e_{n}(t)\right)^{T} \in R^{n}, x_{r}(t)=\left(x_{r 1}(t), x_{r 2}(t), \ldots, x_{r n}(t)\right)^{T} \in R^{n}$, and $x_{r 1}(t)$ is the desired trajectory with

$$
x_{r i}(t)=x_{r 1}^{(i-1)}(t), \quad i \leq n,
$$

where $(\cdot)^{(i-1)}$ denotes the $(i-1)$ th derivative of $(\cdot)$. Then the error dynamic system can be expressed by

$$
\begin{gathered}
\dot{e}_{i}(t)=e_{i+1}(t), \\
\dot{e}_{n}(t)=f(x, t)-x_{r n}(t)+d(t)+b(t) \cdot u(t),
\end{gathered}
$$

where $d(t)=\Delta f(x, t)+p(t)$ is the control disturbance.

The problem to be addressed in this paper is to design a controller such that the tracking error variable satisfies

$$
\lim _{t \rightarrow \infty}\|E(t)\|=\lim _{t \rightarrow \infty}\left\|x(t)-x_{r}(t)\right\| \longrightarrow 0
$$

In this paper, the following lemma is needed

Lemma 2.1 (see [34]). If $w(t): R \rightarrow R$ is a uniformly continuous function for $t \geq 0$ and if

$$
\lim _{t \rightarrow \infty} \int_{0}^{t} w(s) d s
$$

exists and is finite, then

$$
\lim _{t \rightarrow \infty} w(t) \longrightarrow 0
$$

\section{Design of Fuzzy Variable Structure Controller}

In this section, the FVSC method is introduced to realize the control for uncertain system with disturbance. First, the following sliding surface is introduced:

$$
s(t)=C^{T} E(t)=e_{n}(t)+\sum_{i=1}^{n-1} c_{i} e_{i}(t)
$$


where $C=\left[c_{1}, c_{2}, \ldots, c_{n-1}, 1\right]^{T}$ is chosen such that the distribution of the roots of characteristic equation $p^{n-1}+c_{n-1} p^{n-2}+\cdots+c_{2} p+c_{1}=0$ is on the left side of complex plane to make the following system stable:

$$
e_{n}(t)+\sum_{i=1}^{n-1} c_{i} e_{i}(t)=0
$$

Then, we have

$$
\begin{aligned}
\dot{s}(t) & =\dot{e}_{n}(t)+\sum_{i=1}^{n-1} c_{i} e_{i+1}(t) \\
& =f(x)-x_{r n}(t)+d(t)+b(t) \cdot u(t)+\sum_{i=1}^{n-1} c_{i} e_{i+1}(\mathrm{t}) .
\end{aligned}
$$

Based on Lyaponov method and VSC theory, the following theoretical result can be obtained.

First, a fuzzy auxiliary controller $D(t)$ is built to estimate the control disturbance $d(t)$. Corresponding fuzzy rules are given by

$$
\begin{aligned}
& \text { IF } S d(t)>0 \text { THEN } D(t) \text { should be increased, } \\
& \text { IF } S d(t)<0 \text { THEN } D(t) \text { should be decreased, }
\end{aligned}
$$

where

$$
S d(t)=\dot{s}(t)+\varepsilon \cdot \operatorname{sgn}(s(t)) .
$$

The term under consideration $D(t)$ can take a greater value. If it is too big, this may lead to some serious control problem in practice. Therefore in this paper, based on the integral method, the small value $\Delta D(t)$ is recommended to replace by $D(t)$ for their relations as follows:

$$
D(t)=G \int_{0}^{t} \Delta D(s) d s,
$$

where $G$ is the proportionality coefficient.

Let $S d$ denote the fuzzy input $S d(t)$, and $\Delta D$ denotes the fuzzy output $\Delta D(t)$. The fuzzy sets of the input and the output are defined, respectively, as

$$
\begin{aligned}
& S d=\{\mathrm{NB}, \mathrm{NM}, \mathrm{ZO}, \mathrm{PM}, \mathrm{PB}\}, \\
& \Delta D=\{\mathrm{NB}, \mathrm{NM}, \mathrm{ZO}, \mathrm{PM}, \mathrm{PB}\},
\end{aligned}
$$

where NB is negative and large, NM is negative and medium, $\mathrm{ZO}$ is zero, PM is the positive and medium, and $\mathrm{PB}$ is positive and large. 
Select the following fuzzy rules:

R1: IF $S d$ is PB THEN $\Delta D$ is PB,

R2: IF $S d$ is PM THEN $\Delta D$ is PM,

R3: IF $S d$ is ZO THEN $\Delta D$ is $Z O$,

R4: IF $S d$ is NM THEN $\Delta D$ is NM,

R5: IF $S d$ is PB THEN $\Delta D$ is NB.

Hence based on the proposed fuzzy auxiliary controller, the following theoretical result can be concluded.

Theorem 3.1. For $\varepsilon>0$, system (2.1) can track the desired trajectory (2.3) based on the following fuzzy variable structure controller:

$$
u(t)=\frac{1}{b(t)}\left[-f(x)+x_{r n}(t)-\sum_{i=1}^{n-1} c_{i} e_{i+1}(t)-D(t)-\varepsilon \cdot \operatorname{sgn}(s(t))\right]
$$

Proof. Choose the Lyapunov functional candidate as

$$
V(t)=\frac{1}{2} s^{2}(t)
$$

The time derivative of $V(t)$ along trajectories of error model (2.4) is as

$$
\begin{aligned}
\dot{V}(t) & =s(t) \dot{s}(t) \\
& =s(t)\left[f(x)-x_{r n}(t)+d(t)+b(t) \cdot u(t)+\sum_{i=1}^{n-1} c_{i} e_{i+1}(t)\right] .
\end{aligned}
$$

Substituting (3.7) into (3.9), we have

$$
\begin{aligned}
\dot{V}(t) & =s(t)[d(t)-D(t)-\varepsilon \cdot \operatorname{sgn}(s(t))] \\
& =-w(t)
\end{aligned}
$$

where $w(t)=\varepsilon|s(t)|$. For $\varepsilon>0$, we have $\dot{V} \leq 0$. Integrating both sides of (3.9) from 0 to $t$ leads to

$$
\lim _{t \rightarrow \infty} V(t)-V(0) \leq-\lim _{t \rightarrow \infty} \int_{0}^{t} w(s) d s
$$

Since $V(t)$ is positive and $V(0)$ is finite, the following inequality can be concluded:

$$
\lim _{t \rightarrow \infty} \int_{0}^{t} w(s) d s \leq V(0)<\infty
$$




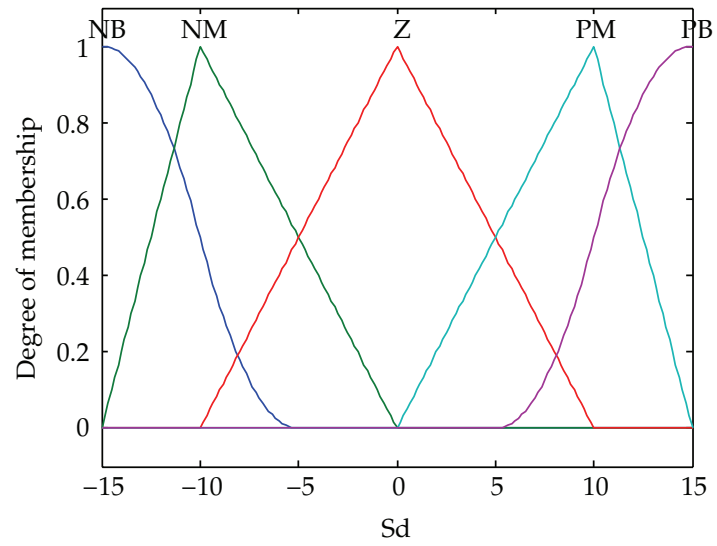

Figure 1: The membership function of the fuzzy input.

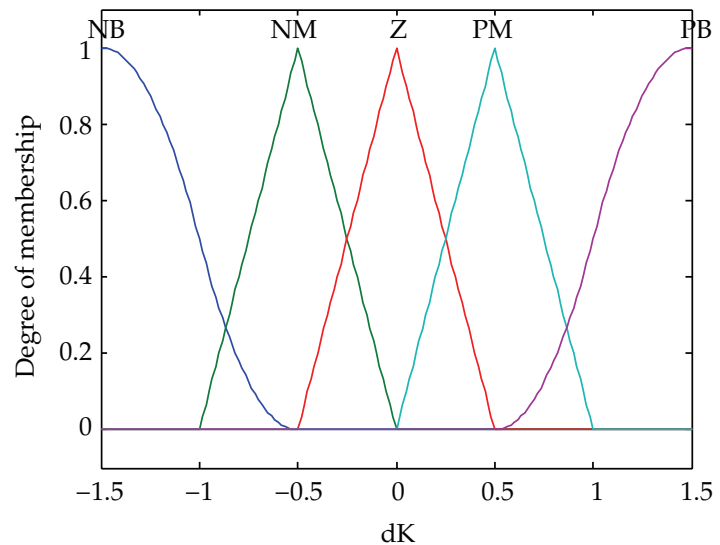

Figure 2: The membership function of the fuzzy output.

Based on Lemma 2.1, we can obtain

$$
\lim _{t \rightarrow \infty} w(t)=\lim _{t \rightarrow \infty} \varepsilon|s(t)| \longrightarrow 0 .
$$

Hence

$$
\lim _{t \rightarrow \infty} E(t) \longrightarrow 0
$$

This means the system control can be achieved based on the fuzzy VSC proposed. The proof of Theorem 3.1 is thus completed.

Remark 3.2. The fuzzy auxiliary controller is constructed based on the feedback signal $S d=$ $\dot{s}(t)+\varepsilon \cdot \operatorname{sgn}(s(t))$, the employed fuzzy rule is simple, and essentially used to keep $S d$ at zero. Hence it can be concluded that $\dot{V}(t)=s(t) \dot{s}(t)=-\varepsilon|s(t)| \leq 0$. This completes our proof. 


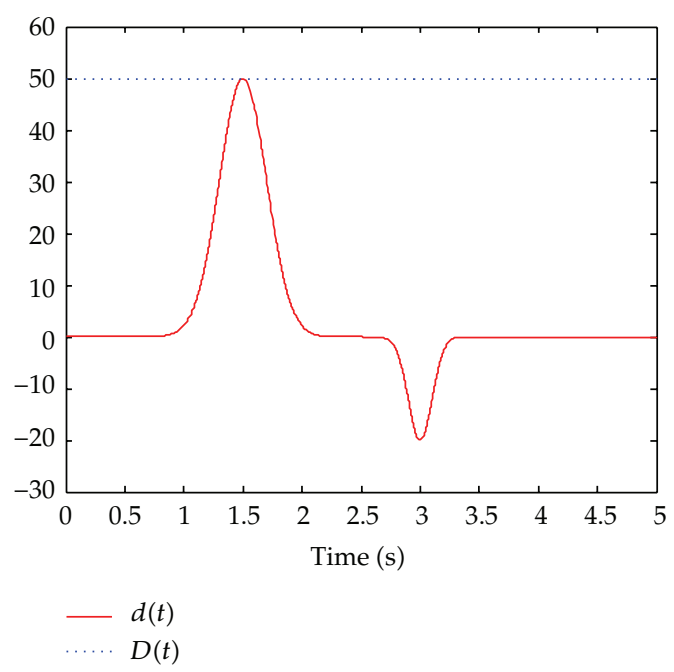

Figure 3: The time response of $d(t)$ and $D(t)$ in case 1 .

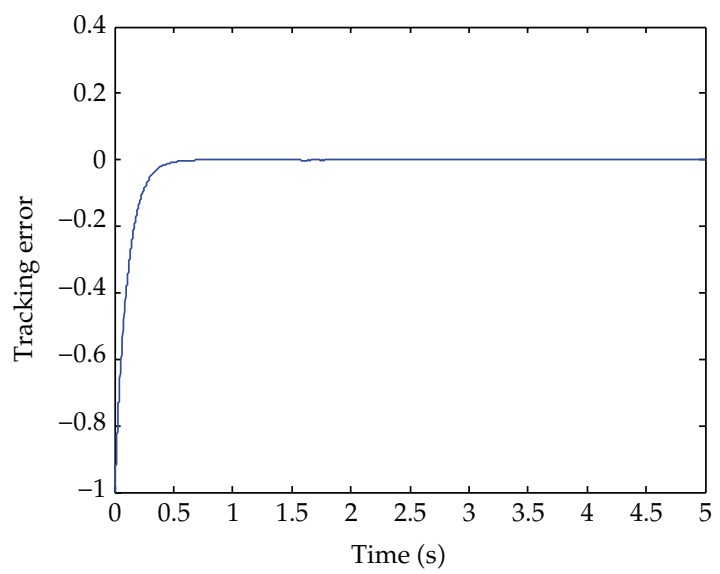

Figure 4: The time response of the tracking error in case 1.

We can see that the fuzzy auxiliary controller and the sliding mode controller come together to realize the effective control on system (2.1).

\section{Numerical Example}

In this section, we will verify the proposed methodology by giving an illustrative example. First consider the following disturbed system

$$
\begin{gathered}
\dot{x}_{1}(t)=x_{2}(t), \\
\dot{x}_{2}(t)=f(x, t)+d(t)+b(t) \cdot u(t),
\end{gathered}
$$




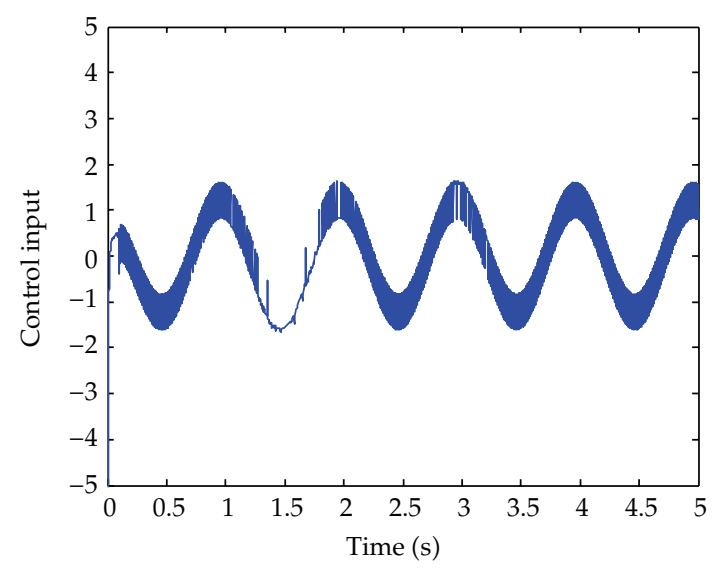

Figure 5: The time response of the control input in case 1.

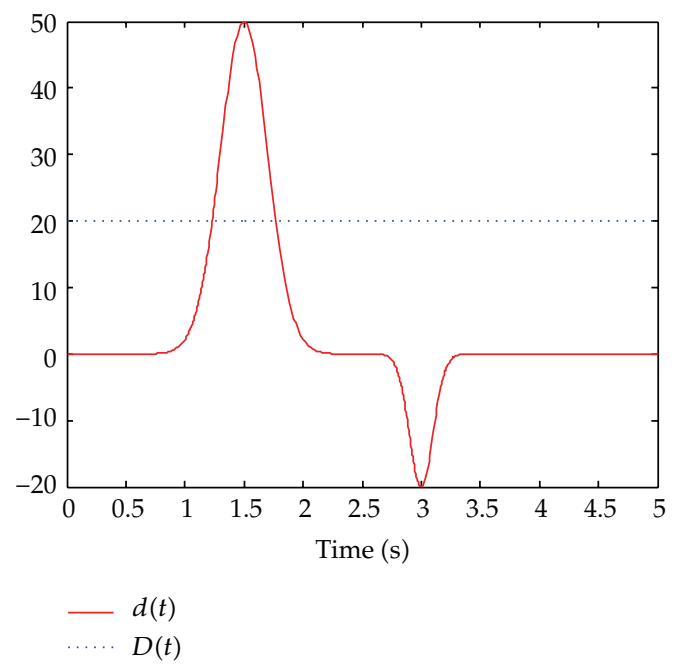

Figure 6: Time response of $d(t)$ and $D(t)$ for case 2 .

where

$$
\begin{aligned}
& f(x, t)=-0.5 x_{2}(t)+x_{1}(t)-x_{1}^{3}(t), \quad b(t)=133, \\
& d(t)=50 \exp \left[-\frac{(t-1.5)^{2}}{2 \cdot 0.2^{2}}\right]-20 \exp \left[-\frac{(t-3)^{2}}{2 \cdot 0.1^{2}}\right] .
\end{aligned}
$$

For simulation purposes, we consider the step size 0.001 second, the initial condition $x_{0}=$ $[-1,-1]^{T}$, the desired trajectory $x_{r}(t)=\sin (2 \pi t)$, and the control parameters $\eta=1.0, r=1.0$, $G=800, c=150$. The membership function of the input and the output of fuzzy system are shown in Figures 1-2. First, we adopt the general VSC method via fixing $D(t)=\max \{|d(t)|\}=$ 50. The simulation results are shown in Figures 3-5. Next, we adopt the general VSC method 


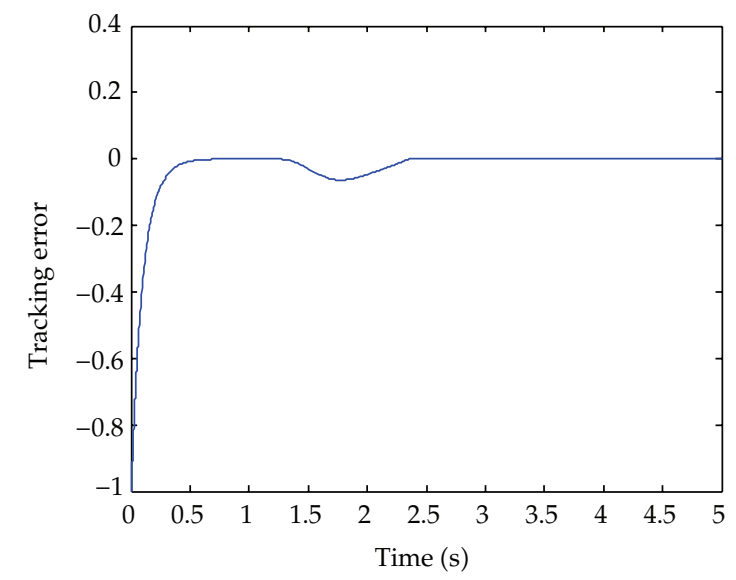

Figure 7: Time response of the tracking error for case 2.

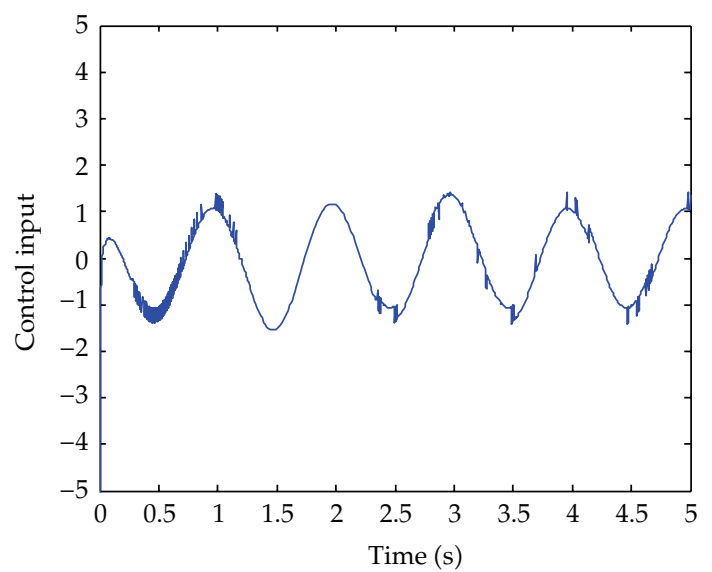

Figure 8: Time response of $u(t)$ for case 2 .

via fixing $D(t)=\max \{|d(t)|\}=20$. The simulation results are shown in Figures 6-8. Finally, we employ the given fuzzy VSC method. The simulation results are shown in Figures 9-11.

Remark 4.1. Figures 3, 6, and 9 show the time response of control disturbance $d(t)$ and its estimate $D(t)$. Figures 4,7 , and 10 show the time response of the tracking error. Figures 5,8 and 11 show the time response of the control input. In case 1, the control disturbance $D(t)$ is fixed at 50 , which is bigger than the upper bound of $d(t)$. From Figures $3-5$ it can be seen, when $d(t) \neq 50$, that there is an obvious chattering phenomenon in control input for the estimation error of $d(t)$. In case 2, the control disturbance $D(t)$ is fixed at 20 , which is less than the upper bound of $d(t)$. From Figures 6-8 it can be seen, when $d(t) \geq 20$, that there exists a big tracking error because the VSC can not be guaranteed at this moment. In case 3 , the control disturbance $d(t)$ is estimated by the fuzzy auxiliary controller. From Figures 9-11 it can be seen that the control for the given system is realized within 1 second and the chattering phenomenon is reduced distinctly, which demonstrates the effectiveness of the presented fuzzy VSC method. 


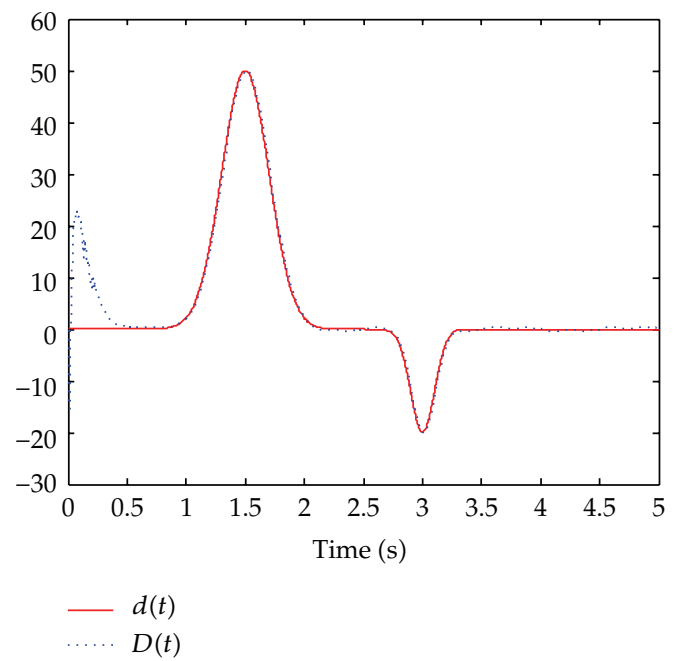

Figure 9: The time response of $d(t)$ and $D(t)$ in case 3 .

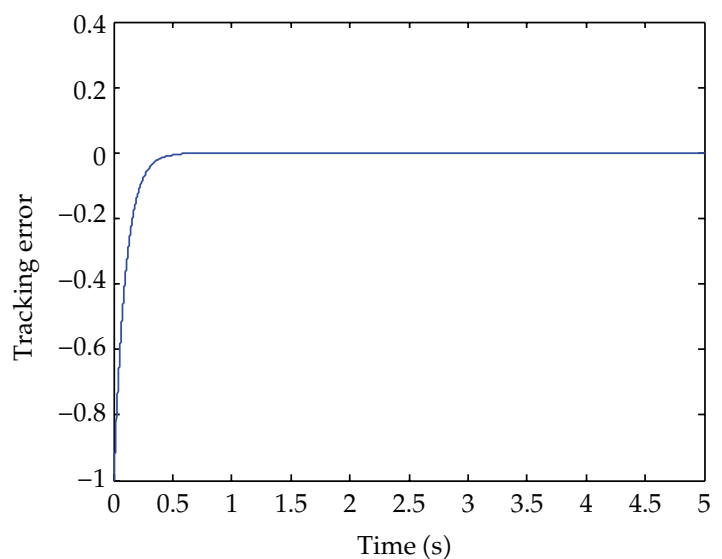

Figure 10: The time response of the tracking error in case 3.

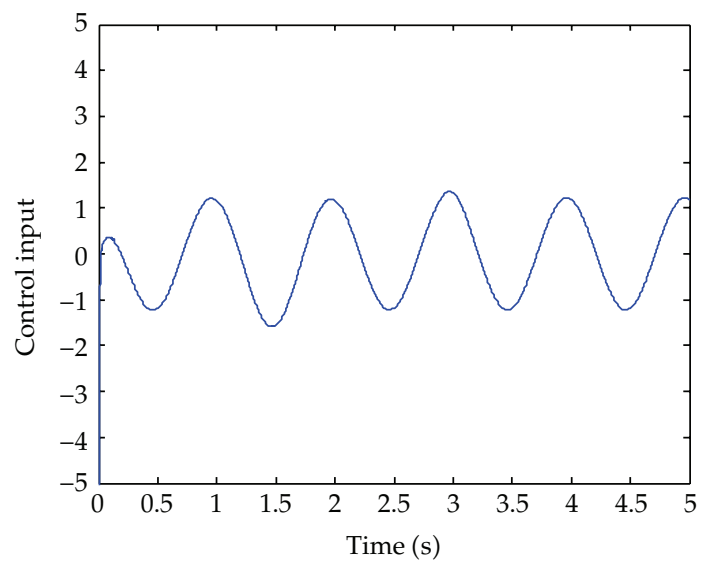

Figure 11: The time response of the control input in case 3. 
Remark 4.2. From the simulation results, we can see that the chattering phenomenon is reduced effectively by using the proposed fuzzy controller however there still exists the switching term $\varepsilon \cdot \operatorname{sgn}(s(t))$ in control signal although $\varepsilon$ is a small constant. To further overcome the control chattering phenomenon, the switching term $\varepsilon \cdot \operatorname{sgn}(s(t))$ is recommended to be substituted for $\varepsilon \cdot \operatorname{sat}(s(t))$.

\section{Conclusion}

In this paper, the fuzzy variable structure control problem has been studied. The fuzzy control method and the switching control method have been employed to realize the control for uncertain system with disturbance, they possess the characteristic of simpleness in design and effectiveness in attenuating the control chattering, and aresuitable for the application in engineering. Some typical numerical examples have been included afterward to demonstrate the effectiveness of the given controller.

\section{Acknowledgments}

This work was partially supported by the Key Projects of Xihua University (Z1120946), the National Key Basic Research Program (973), China (no. 2012CB215202), the 111 Project (B12018), the National Natural Science Foundation of China (nos. 61174058, and 61170030), and the Engineering and Physical Sciences Research Council, UK (EP/F029195).

\section{References}

[1] V. I. Utkin, "Variable structure systems with sliding modes," IEEE Transactions on Automatic Control, vol. 22, no. 2, pp. 212-222, 1977.

[2] H. H. Choi, "Adaptive controller design for uncertain fuzzy systems using variable structure control approach," Automatica, vol. 45, no. 11, pp. 2646-2650, 2009.

[3] J. P. V. S. Cunha, R. R. Costa, F. Lizarralde, and L. Hsu, "Peaking free variable structure control of uncertain linear systems based on a high-gain observer," Automatica, vol. 45, no. 5, pp. 1156-1164, 2009.

[4] H. Xing, C. C. Gao, and D. Li, "Sliding mode variable structure control for parameter uncertain stochastic systems with time-varying delay," Journal of Mathematical Analysis and Applications, vol. 355, no. 2, pp. 689-699, 2009.

[5] X. Chen, T. Hisayama, and C. Y. Su, "Pseudo-inverse-based adaptive control for uncertain discrete time systems preceded by hysteresis," Automatica, vol. 45, no. 2, pp. 469-476, 2009.

[6] L. Wu, D. W. C. Ho, and C. W. Li, "Sliding mode control of switched hybrid systems with stochastic perturbation," Systems and Control Letters, vol. 60, no. 8, pp. 531-539, 2011.

[7] Z. Lin, Y. Xia, P. Shi, and H. Wu, "Robust sliding mode control for uncertain linear discrete systems independent of time-delay," International Journal of Innovative Computing, Information and Control, vol. 7, no. 2, pp. 869-880, 2011.

[8] T. E. Lee, J. P. Su, K. W. Yu, and K. H. Hsia, "Multi-objective fuzzy optimal design of alpha-beta estimators for nonlinear variable structure control," International Journal of Innovative Computing, Information and Control, vol. 7, no. 5, pp. 2123-2140, 2011.

[9] S. Qu, Z. Lei, Q. Zhu, and H. Nouri, "Stabilization for a class of uncertain multi-time delays system using sliding mode controller," International Journal of Innovative Computing, Information and Control, vol. 7, no. 7, pp. 4195-4205, 2011.

[10] Y. Niu and D. W. C. Ho, "Stabilization of discrete-time stochastic systems via sliding mode technique," Journal of the Franklin Institute, vol. 349, pp. 1497-1508, 2012.

[11] T. Jia, Y. Niu, and Y. Zou, "Sliding mode control for stochastic systems subject to packet losses," Information Sciences, vol. 217, pp. 117-126, 2012. 
[12] L. Wu and D. W. C. Ho, "Sliding mode control of singular stochastic hybrid systems," Automatica, vol. 46, no. 4, pp. 779-783, 2010.

[13] H. R. Karimi, "A sliding mode approach to $H_{\infty}$ synchronization of master-slave time-delay systems with Markovian jumping parameters and nonlinear uncertainties," Journal of the Franklin Institute, vol. 349, no. 4, pp. 1480-1496, 2012.

[14] L. Wu, P. Shi, and H. Gao, "State estimation and sliding-mode control of markovian jump singular systems," IEEE Transactions on Automatic Control, vol. 55, no. 5, pp. 1213-1219, 2010.

[15] B. Jiang, P. Shi, and Z. Mao, "Sliding mode observer-based fault estimation for nonlinear networked control systems," Circuits, Systems, and Signal Processing, vol. 30, no. 1, pp. 1-16, 2011.

[16] M. Liu, P. Shi, L. Zhang, and X. Zhao, "Fault tolerant control for nonlinear Markovian jump systems via proportional and derivative sliding mode observer technique," IEEE Transactions on Circuits and Systems, vol. 58, pp. 2755-2764, 2011.

[17] H. R. Karimi, "Robust synchronization and fault detection of uncertain master-slave systems with mixed time-varying delays and nonlinear perturbations," International Journal of Control, Automation and Systems, vol. 9, no. 4, pp. 671-680, 2011.

[18] H. R. Karimi, "Adaptive $H_{\infty}$ synchronization problem of uncertain master-slave systems with mixed time-varying delays and nonlinear perturbations: an LMI approach," International Journal of Automation and Computing, vol. 8, no. 4, pp. 381-390, 2011.

[19] H. R. Karimi, M. Zapateiro, and N. Luo, "Stability analysis and control synthesis of neutral systems with time-varying delays and nonlinear uncertainties," Chaos, Solitons and Fractals, vol. 42, no. 1, pp. 595-603, 2009.

[20] H. R. Karimi and P. Maass, "Delay-range-dependent exponential $H_{\infty}$ synchronization of a class of delayed neural networks," Chaos, Solitons and Fractals, vol. 41, no. 3, pp. 1125-1135, 2009.

[21] H. R. Karimi, B. Lohmann, B. Moshiri, and P. J. Maralani, "Wavelet-based identification and control design for a class of nonlinear systems," International Journal of Wavelets, Multiresolution and Information Processing, vol. 4, no. 1, pp. 213-226, 2006.

[22] H. R. Karimi and H. Gao, "Mixed $\mathrm{H}_{2} / \mathrm{H}_{\infty}$ output-feedback control of second-order neutral systems with time-varying state and input delays," ISA Transactions, vol. 47, no. 3, pp. 311-324, 2008.

[23] H. Yang, P. Shi, J. Zhang, and J. Qiu, "Robust $H_{\infty}$ control for a class of discrete time fuzzy systems via delta operator approach," Information Sciences, vol. 184, no. 1, pp. 230-245, 2012.

[24] Z. Wu, P. Shi, H. Su, and J. Chu, "Reliable $H_{\infty}$ control for discrete-time fuzzy systems with infinitedistributed delay," IEEE Transactions on Fuzzy Systems, vol. 20, no. 1, pp. 22-31, 2012.

[25] Q. Zhou, P. Shi, J. Lu, and S. Xu, "Adaptive output feedback fuzzy tracking control for a class of nonlinear systems," IEEE Transactions on Fuzzy Systems, vol. 19, no. 5, pp. 972-982, 2011.

[26] X. Su, P. Shi, and L. Wu, "A novel approach to filter design for T-S fuzzy discrete-time systems with time-varying delay," IEEE Transactions on Fuzzy Systems. In press.

[27] Z. Gao, B. Jiang, P. Shi, and Y. Xu, "Fault accommodation for near space vehicle attitude dynamics via T-S fuzzy models," International Journal of Innovative Computing, Information and Control, vol. 6, no. 11, pp. 4843-4856, 2010.

[28] Z. Pei and P. Shi, "Fuzzy risk analysis based on linguistic aggregation operations," International Journal of Innovative Computing Information and Control, vol. 7, no. 12, pp. 7105-7117, 2011.

[29] G. Wang, P. Shi, and C. Wen, "Fuzzy approximation relations on fuzzy n-cell number space and their applications in classification," Information Sciences, vol. 181, no. 18, pp. 3846-3860, 2011.

[30] J. Zhang, P. Shi, and Y. Xia, "Robust adaptive sliding-mode control for fuzzy systems with mismatched uncertainties," IEEE Transactions on Fuzzy Systems, vol. 18, no. 4, pp. 700-711, 2010.

[31] Q. Zhou, P. Shi, S. Xu, and H. Li, "Adaptive output feedback control for nonlinear time-delay systems by fuzzy approximation approach," IEEE Transactions on Fuzzy Systems. In press.

[32] X. Zhao, Y. Xu, Z. Zhang, and P. Shi, "Design of PSO fuzzy neural network control for ball and plate system," International Journal of Innovative Computing Information and Control, vol. 7, no. 12, pp. 70917103, 2011.

[33] K. Zhang, B. Jiang, and P. Shi, "Fault estimation observer design for discrete-time Takagi-Sugeno fuzzy systems based on piecewise Lyapunov functions," IEEE Transactions on Fuzzy systems, vol. 20, no. 1, pp. 192-200, 2012.

[34] H. Khalil, Nonlinear Systems, Macmillan, New York, NY, USA, 1992. 


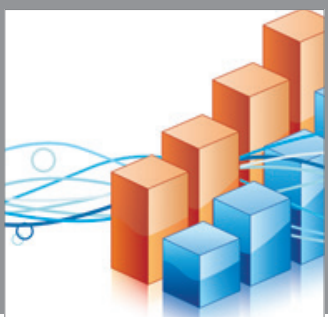

Advances in

Operations Research

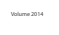

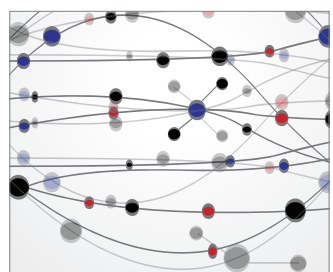

\section{The Scientific} World Journal
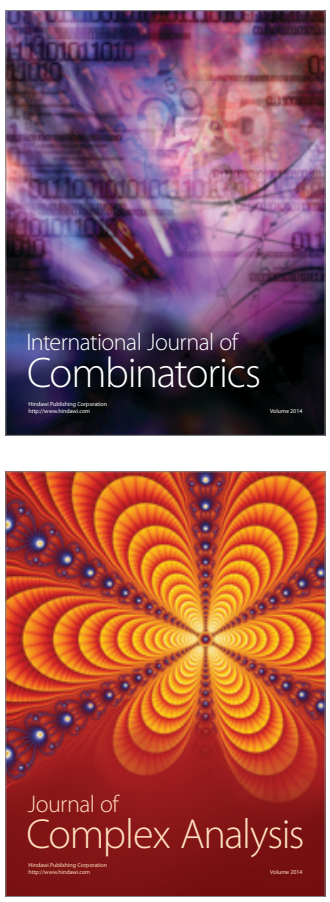

International Journal of

Mathematics and

Mathematical

Sciences
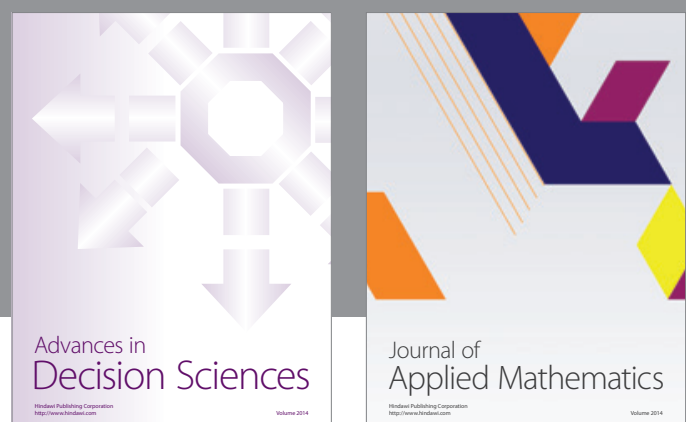

Journal of

Applied Mathematics
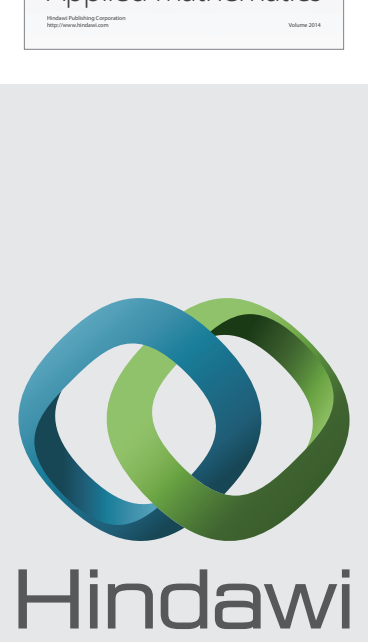

Submit your manuscripts at http://www.hindawi.com
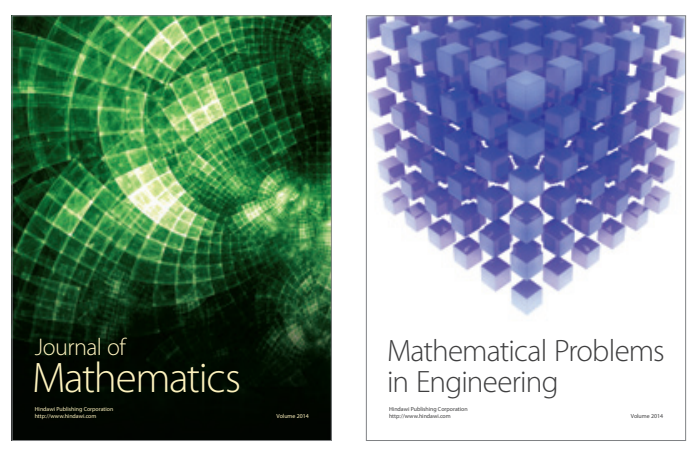

Mathematical Problems in Engineering
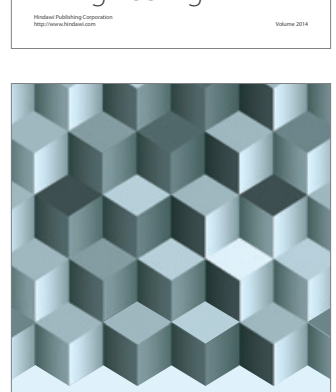

Journal of

Function Spaces
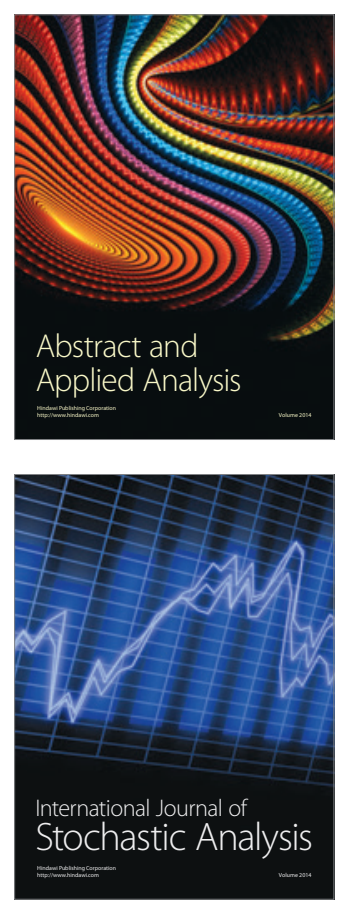

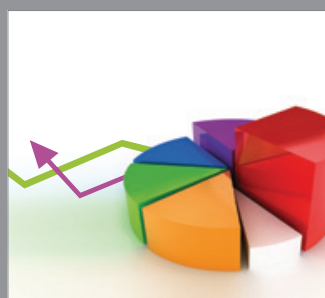

ournal of

Probability and Statistics

Promensencen
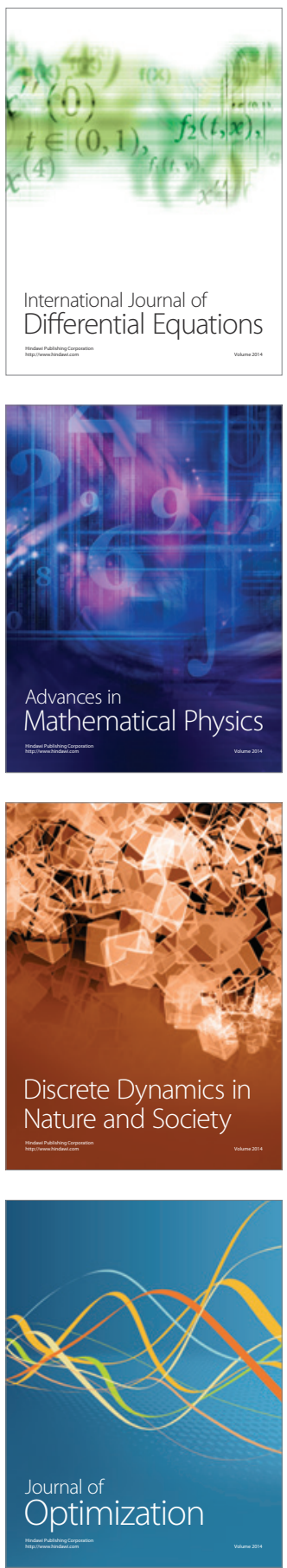